Quim. Nova, Vol. 35, No. 6, 1276-1280, 2012

\title{
IMPLANTAÇÃO E IMPLEMENTAÇÃO DAS NORMAS DAS BOAS PRÁTICAS LABORATORIAIS (BPL) NO LABORATÓRIO DE ANÁLISES DE RESÍDUOS DA UNIVERSIDADE ESTADUAL DE CAMPINAS
}

\author{
Nadia Regina Rodrigues*, Ana Paula Ferreira de Souza e Marcelo Watanabe \\ Universidade Estadual de Campinas, Cidade Universitária, C. P. 6171, 13081-970 Campinas - SP, Brasil
}

Recebido em 13/6/11; aceito em 16/12/11; publicado na web em 23/3/12

\begin{abstract}
IMPLANTATION AND IMPLEMENTATION OF STANDARDS OF GOOD LABORATORY PRACTICE (GLP) AT THE LABORATORY FOR RESIDUE ANALYSIS OF THE STATE UNIVERSITY OF CAMPINAS. In the context of quality and good laboratory practices, the article recovers some historical data. From a specific Institutional situation (CPQBA/UNICAMP), is presented an experience of establishing and implementing a standard (NIT-DICLA-035) for good laboratory practice according to definitions of the Brazilian authority (INMETRO) responsible for regulating, monitoring, supervising and recognition in this area. The issue aims to focus on studies of pesticide residues in GLP parameters.
\end{abstract}

Keywords: GLP; quality; recognition.

\section{INTRODUÇÃO}

A confiabilidade dos estudos efetuados sob as normas de BPL utilizados para registro de produtos fornece maior segurança, principalmente no que tange à análise de risco decorrente da utilização dos mesmos, proporcionando impactos positivos na preservação da saúde da população.

A Food and Drug Administration (FDA) visando a segurança dos dados recebidos em seus estudos, regulamentou e publicou as normas de Boas Práticas Laboratoriais nos Estados Unidos da América. Essa mesma ação foi repetida pela Organization for Economic Cooperation and Development (OECD) que, em 1981, publicou uma série de parâmetros norteadores das BPL e pela Environmental Protection Agency (EPA) em 1980. ${ }^{1}$

No Brasil as implementações das BPL tiveram início, em 1994, com a exigência do Instituto Brasileiro do Meio Ambiente e dos Recursos Naturais Renováveis (IBAMA), para aceitação dos laudos ecotoxicológicos emitidos por laboratórios envolvidos nos estudos sobre o potencial de periculosidade ambiental de produtos agrotóxicos. ${ }^{2}$

Em 1997, o IBAMA em conjunto com o Instituto Nacional de Metrologia Qualidade e Tecnologia (INMETRO) publicou uma portaria estabelecendo os critérios de BPL para o credenciamento feito pelo INMETRO. Essa portaria abrange todos os laboratórios (incluindo os estrangeiros) que realizam estudos físico-químicos, toxicológicos e ecotoxicológicos na avaliação do impacto ambiental de produtos químicos, bioquímicos e biotecnológicos. Como parte das ações tomadas para essa demanda, o INMETRO publicou o documento "Princípios das Boas Práticas de Laboratório", tendo como base o documento "OECD Series on Principles of Good Laboratory Practice". Concomitantemente, por meio da Coordenação Geral de Acreditação (Cgcre), o INMETRO baixou uma normativa específica para BPL, denominada Norma Interna Técnica da Divisão de Acreditação de Laboratórios, NIT-DICLA-035. ${ }^{3}$

Essa Norma se aplica a todos os testes não clínicos de avaliação do risco ambiental e à saúde humana dos produtos agrotóxicos, seus componentes e afins, produtos farmacêuticos, cosméticos, preservativos de madeira, aditivos de alimentos e de rações, produtos veterinários, domissanitários, produtos químicos industriais, organismos geneticamente modificados e remediadores.

\footnotetext{
*e-mail: nadia @cpqba.unicamp.br
}

Em março de 2011, o Brasil foi inserido de forma plena aos Atos do Conselho da OECD relacionados à Aceitação Mútua de Dados (MAD) para avaliação de substâncias químicas. Essa inclusão permite que os testes realizados em laboratórios brasileiros reconhecidos pelo INMETRO/Cgcre sejam aceitos nos países membros da OECD.

Os benefícios da inclusão do Brasil incluem a dinamização no processo das exportações brasileiras, reduzindo assim as barreiras técnicas que os produtos de exportação brasileiros sofrem constantemente. Outro ganho diz respeito à possibilidade que os estudos realizados pelas empresas para fins de registro de determinados produtos sejam conduzidos em laboratórios brasileiros, uma vez que esses registros são aceitos em países da Europa e nos EUA.

No âmbito universitário existe uma ampla discussão sobre a aplicabilidade dos princípios de BPL, isto porque a acreditação é concedida contra uma determinada agenda de avaliações. Essas avaliações são, além de minuciosas, onerosas, o que acaba por afastar essa acreditação do cenário da universidade. No entanto, o uso dos conceitos de BPL pelas universidades pode auxiliar na qualidade dos dados gerados. Os princípios não precisam ser implementados na sua totalidade, mas podem nortear as pesquisas. Em teoria, a pesquisa e o desenvolvimento, consistindo em medições objetivas não rotineiras, se devidamente documentados e validados, podem ser credenciados, desde que o laboratório considere pertinente. ${ }^{4} \mathrm{Em}$ consequência, a pesquisa ganha com essas ações, obtendo maior transparência e integridade dos dados por meio da documentação adequada. Vale ressaltar que avaliações externas podem ser úteis na demonstração da qualidade a clientes, órgãos reguladores, organismos de financiamento ou mesmo para comparar com outros o seu nível de qualidade a fim de fazer melhorias. ${ }^{1}$ Recentemente esse ganho para a pesquisa foi enfatizado pela Fundação de Amparo à Pesquisa do Estado de São Paulo (FAPESP) que lançou o Código de Boas Práticas Científicas. ${ }^{5}$

Assim, com a finalidade de estabelecer convênios com empresas do setor de agrotóxicos, atendendo à exigência da Agência Nacional de Vigilância Sanitária (ANVISA), conforme Resolução RDC 216 de 15 de dezembro de 2006, ${ }^{6}$ a Divisão de Análises de Resíduos do Centro Pluridisciplinar de Pesquisas Químicas, Biológicas e Agrícolas (CPQBA) da Universidade Estadual de Campinas (UNICAMP) em 2010 cumpriu todos os requisitos e foi reconhecida pelo INMETRO em BPL. 


\section{METODOLOGIA}

\section{Implantação de BPL}

\section{Conscientização do pessoal}

O sucesso da implantação de um sistema de gestão em BPL requer não só o compromisso de toda a equipe; passa por uma mudança de postura na execução das atividades em um laboratório. Portanto, os contatos iniciais da equipe com as normas a serem adotadas, a compreensão das mesmas e a elaboração de uma estratégia com prioridades constituem a primeira etapa. Para isso organizou-se um curso para a sensibilização de todas as pessoas envolvidas direta ou indiretamente na implantação das BPL. Essa oportunidade foi disponibilizada para todos, desde a diretoria até os técnicos de apoio.

\section{Instalações}

O laboratório, inaugurado em setembro de 2006, foi planejado e construído de maneira a atender as BPL. No caso do laboratório em questão, que analisa traços de produtos, houve o cuidado de se minimizar contaminações. Cada atividade distinta é realizada em salas específicas. A área administrativa fica completamente isolada da área dos laboratórios

\section{Nomeação para cargos BPL}

Atendendo à norma NIT-Dicla-035, foram estabelecidas as responsabilidades dos integrantes do laboratório e distribuídas de acordo com o organograma apresentado na Figura 1

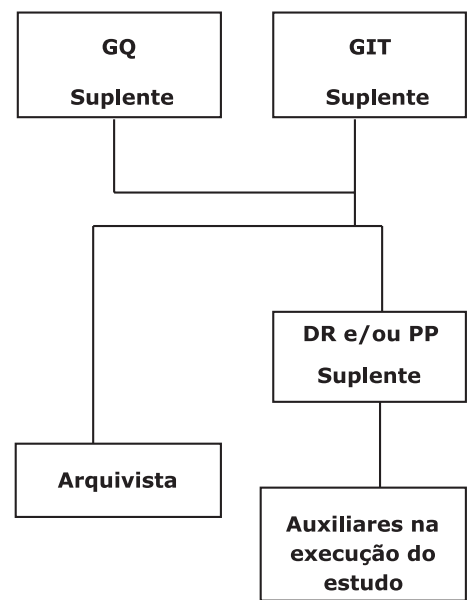

Figura 1. Organograma BPL. GIT Gerente da Instalação de Teste-assegura que os princípios da BPL sejam cumpridos no laboratório. $G Q$ Garantia da Qualidade - pessoa designada, desvinculada da condução do estudo e responsável por garantir a gestão da Instalação Teste em conformidade com os princípios de BPL. DR Diretor de Estudo - responsável pela condução e relatório final do estudo. PP Pesquisador Principal - assegura que a fase da qual ele é executor seja conduzida de acordo com os princípios de BPL. Arquivista - responsável pela guarda dos documentos

\section{Materiais e calibração de equipamentos}

Todos os equipamentos e materiais envolvidos na BPL, além de separados e devidamente identificados, são periodicamente inspecionados, limpos e submetidos à manutenção. Os que necessitam de calibração possuem um programa definido. As calibrações são sempre executadas por empresas credenciadas.

\section{Definição de protocolos}

De acordo com os regulamentos e diretrizes de BPL, os procedimentos operacionais padrão (POPs) são um componente exigido em um programa de conformidade numa unidade operacional. Quando convenientemente desenvolvidos e seguidos, os POPs asseguram consistência e boa definição a um programa de pesquisa, independentemente de quem conduz o trabalho. Esses procedimentos devem ser tanto de caráter administrativo como técnico. Toda a sistemática decorrente das atividades do laboratório deve ser descrita de forma objetiva, concisa e autoexplicativa. Os itens que devem constar num procedimento técnico ou administrativo estão detalhados na Tabela 1.

Vinculada a qualquer procedimento, é importante a criação de formulários de registro para que sejam anotados dados e atividades. ${ }^{7}$ Estes são um componente do sistema de BPL com características mais simples do que os POPs e seus itens estão descritos na Tabela 2.

\section{Procedimentos administrativos}

Os procedimentos administrativos (PAs) foram elaborados de modo a instruir as pessoas envolvidas como elaborar um POP, um plano de estudo (PE) e a agenda mestra. Foram elaborados PAs que incluem como evidenciar a comunicação interna, revisar o PE, inspecionar instalações e fases críticas, realizar auditoria de dados e revisão de relatórios, proceder com ações corretivas e preventivas, utilizar o selo de reconhecimento em BPL e realizar treinamento de pessoal.

\section{Procedimentos técnicos}

Foram elaborados procedimentos técnicos de metodologia (PTM) para determinação de resíduos de princípios ativos, validação de método para análise de resíduo, controle de qualidade analítica, manuseio de amostras de sistemas testes, processamento de amostras, lavagem de vidraria, descartes de solventes, sistemas informatizados e alguns outros. Vinculados a esses procedimentos foram criados formulários para registro de dados, da cadeia de custódia, do processamento de amostras, da avaliação da substância de referência, da análise crítica das condições do sistema teste, do controle de sistema teste, de dados brutos de análise de resíduos etc.

Foram elaborados procedimentos técnicos de operação de equipamentos (PTE) para balanças analíticas, deionizador de água, centrífuga refrigerada, triturador de gelo, bomba de alta pressão, detectores de cromatógrafos etc. Vinculados a esses procedimentos foram criados formulários para registro de uso e de calibrações, quando pertinentes, desses equipamentos.

\section{RESULTADOS}

\section{Implementação de BPL}

O Laboratório de Resíduos do CPQBA/UNICAMP foi capaz de atender as exigências, descritas na NIT DICLA 035, para infraestrutura, gerenciamento, pessoal, garantia e controle de qualidade, documentação dos resultados e dados brutos. Esses itens são considerados pré-requisitos para a obtenção de resultados confiáveis e rastreáveis. A implementação desses se volta à dinâmica funcional no laboratório, que pode ser considerada em três partes inter-relacionadas: o analista, a infraestrutura básica e a análise propriamente dita.

\section{$O$ analista}

A análise de resíduos consiste numa sequência de procedimentos, a maior parte dos quais são compreendidos por uma pessoa treinada. No entanto, as análises podem ser desafiantes, sendo essencial a atenção para cada detalhe, uma vez que as concentrações do analito são muito baixas no intervalo de $\mu \mathrm{g} \mathrm{kg}^{-1} \mathrm{a} \mathrm{mg} \mathrm{kg}{ }^{-1}$, e não é incomum a ocorrência de perdas, interferências e contaminações. Assim, o pessoal do CPQBA foi qualificado e treinado no uso correto de equipamentos e nas habilidades adequadas ao laboratório. Cada procedimento do laboratório englobou um item que comprovou a 
Tabela 1. Elementos contemplados na elaboração de procedimentos técnicos e administrativos

\begin{tabular}{|c|c|}
\hline Campos & Descrições \\
\hline$\underline{\text { Título }}$ & Deve ser preciso e conciso, indicando sem ambiguidade o assunto tratado pelo procedimento. \\
\hline Emissão & Sigla do departamento que emitiu o procedimento. \\
\hline Edição/Revisão & $\begin{array}{l}\text { Indica o número da edição em que ocorreram alterações que resultem em grandes mudanças textuais e o número } \\
\text { da revisão em que ocorreram pequenas alterações. }\end{array}$ \\
\hline Número de páginas & Número de páginas do documento. \\
\hline Objetivos & $\begin{array}{l}\text { Deve definir, sem ambiguidade, o assunto do procedimento e os aspectos abrangidos pelo mesmo. Seu campo e } \\
\text { limites de aplicabilidade podem formar parte deste item ou serem definidos em separado. }\end{array}$ \\
\hline Amplitude & $\begin{array}{l}\text { Define as estruturas organizacionais, procedimentos, pessoas, atividades, serviços e/ou tipos de elementos (amostras, } \\
\text { equipamentos, produtos etc.) aos quais se aplica o procedimento, assim como os limites de sua aplicabilidade. } \\
\text { Não deve conter requisitos. }\end{array}$ \\
\hline Referências & $\begin{array}{l}\text { Inclui uma lista completa dos documentos normativos ou descritivos, referenciados no texto, que são indispensáveis } \\
\text { ao cumprimento do procedimento. }\end{array}$ \\
\hline Responsabilidade da aplicação & $\begin{array}{l}\text { Deve ser indicado de forma clara o pessoal ou a subunidade organizacional responsável pela correta aplicação } \\
\text { do procedimento. }\end{array}$ \\
\hline Descrição & $\begin{array}{l}\text { Fornece todas as instruções específicas e detalhadas referentes ao procedimento. Seu conteúdo deve ser organizado } \\
\text { passo a passo, de acordo com a sequência do procedimento e conter toda a informação necessária para garantir a } \\
\text { reprodutibilidade do processo e dos resultados. } \\
\text { Deve descrever os seguintes itens: } \\
\text { Princípios - indicar os conceitos químicos e/ou físicos nos quais o procedimento a ser seguido está baseado. } \\
\text { Reagentes e Materiais - descrever todos os materiais e/ou reagentes necessários para a realização da análise. } \\
\text { Quando aplicável, descrever as especificações. } \\
\text { Equipamentos - quando aplicável, indicar os equipamentos a serem usados. } \\
\text { Preparo da amostra - quando aplicável, descrever tratamento inicial da amostra. } \\
\text { Procedimento - descrever detalhadamente o procedimento a ser seguido para a realização do objetivo. Iniciar frases } \\
\text { sempre no infinitivo. Por ex: Transferir para um balão volumétrico. } \\
\text { Expressão de resultados - indicar a forma como os resultados serão expressos. } \\
\text { Relatório de análise - Deve descrever de forma geral o método usado, indicando o procedimento seguido (PTM } \\
\text { XX XXX), as amostras analisadas, as massas e diluições, os padrões, os equipamentos, os dados brutos, fórmulas } \\
\text { dos cálculos, os resultados finais como descrito no item Expressões de Resultados, o(s) analista(s), o pesquisador } \\
\text { principal e o responsável pela GQ. }\end{array}$ \\
\hline Data de efetivação & Data em que o documento entrou em vigor dentro do laboratório. \\
\hline Responsabilidade & $\begin{array}{l}\text { Chama a atenção para o usuário seguir exatamente o procedimento descrito. Qualquer dúvida, anormalidade ou } \\
\text { sugestão deve ser levada ao conhecimento do gerente da Instalação Teste. }\end{array}$ \\
\hline $\begin{array}{l}\text { Assinatura do pessoal habilitado para a } \\
\text { aplicação do procedimento }\end{array}$ & $\begin{array}{l}\text { Assinatura da pessoa treinada, sua função, data do treinamento e rubrica do supervisor. A rubrica do supervisor } \\
\text { confirma a aptidão da pessoa em executar o procedimento. }\end{array}$ \\
\hline Definições e siglas & Item opcional que relaciona as siglas e abreviaturas utilizadas no texto. \\
\hline Arquivo & $\begin{array}{l}\text { Descreve a distribuição das cópias controladas do procedimento, eventuais restrições à distribuição, assim como } \\
\text { a localização do arquivo do mesmo. }\end{array}$ \\
\hline Elaboração & Nome e assinatura do responsável pela elaboração do documento. \\
\hline Verificação/Aprovação & Assinatura do responsável por verificar e aprovar o conteúdo do documento. \\
\hline
\end{tabular}

habilitação do pessoal envolvido. As pessoas depois de treinadas entenderam os objetivos de cada etapa do método, a importância de seguir exatamente o que está descrito e anotar qualquer desvio inevitável. Também foram treinadas na avaliação e interpretação dos dados. Esses aspectos foram conseguidos em quatro fases:

Fase 1 - Leitura dos procedimentos técnicos (PTM, PTE) e administrativos $(\mathrm{PA})$ referentes às atividades a serem desenvolvidas.

Fase 2 - Acompanhamento da execução de um procedimento.

Fase 3 - Realização do procedimento ou método pelo menos três vezes.

Fase 4 - Habilitação quando os resultados obtidos se mostraram compatíveis com o padrão de aceitabilidade adotado no laboratório.

\section{As análises}

Uma diferença significativa entre uma análise de traços e uma macroanálise é a possível ocorrência de contaminação ou interferência naquela análise. Mesmo uma pequena contaminação na amostra final pode causar erros nos resultados como falso positivo, falso negativo ou perda de sensibilidade que impedirá os resíduos de serem detectados. Uma contaminação pode surgir de quase tudo o que é usado ou pode estar associada com amostragem, transporte, armazenamento ou análise. A verificação de possíveis interferências resultantes de vidraria, reagentes e solventes é efetuada com a análise de um branco dos reagentes PTM (Controle de Qualidade Analítica).

A análise de princípios ativos de pesticidas em alimentos, água e solo no nosso laboratório é enfocada em quatro grandes grupos, com ênfase na preservação da integridade das amostras (recebimento, preparo e armazenamento das amostras); na análise propriamente dita (extração, limpeza de extrato, determinação do ingrediente ativo), com garantia da qualidade dos métodos utilizados (validação de metodologia) e na preservação dos dados gerados (dados brutos e relatório). No Laboratório do CPQBA foram gerados, para garantir a execução das análises segundo a BPL, 164 documentos. Em números específicos, têm-se 15 anexos, 14 PA, 27 PTM, 58 PTE e 50 formulários.

Para exemplificar a implementação das normas de BPL no laboratório, apresentamos a seguir um caso de análise de resíduos de um herbicida em grãos de soja. Para a execução desse estudo foram gerados 126 documentos, distribuídos como mostra a Figura 2.

Na primeira etapa (recebimento das amostras) foram utilizados 3 PA (inspeção de fase crítica, treinamento de pessoal e comunicação interna), 4 formulários de registros (cadeia de custódia, análise crítica das condições do sistema teste, controle do sistema teste e registro 
Tabela 2. Elementos contemplados na elaboração de formulários de registro

\begin{tabular}{ll}
\hline Campos & Descrições \\
\hline Título & $\begin{array}{l}\text { Deve constar a palavra Formulário antes do } \\
\text { assunto a que se refere. }\end{array}$ \\
\hline Emissão & $\begin{array}{l}\text { Sigla do departamento que emitiu o pro- } \\
\text { cedimento. }\end{array}$ \\
\hline Edição/Revisão & $\begin{array}{l}\text { Indica o número da edição em que ocorreram } \\
\text { alterações que resultem em grandes mudanças } \\
\text { textuais e o número da revisão em que ocor- } \\
\text { reram pequenas alterações. }\end{array}$ \\
\hline Número de páginas & $\begin{array}{l}\text { Indicação da página, preferencialmente no } \\
\text { rodapé ao centro. }\end{array}$ \\
\hline Referências & $\begin{array}{l}\text { Deve indicar o(s) procedimento(s) técnico(s) } \\
\text { ou administrativo(s) ao(s) qual(is) o formu- } \\
\text { lário está vinculado. }\end{array}$ \\
\hline Descrição & $\begin{array}{l}\text { Fornece todos os campos que são essenciais } \\
\text { à execução de um procedimento ao qual o } \\
\text { formulário se vincula. }\end{array}$ \\
\hline Data de preenchimento & Data(s) em que o formulário foi preenchido. \\
\hline Responsabilidade & $\begin{array}{l}\text { Nome da pessoa responsável pelo preenchi- } \\
\text { mento. }\end{array}$ \\
\hline Arquivo & $\begin{array}{l}\text { Descreve o local onde deve ser anexado } \\
\text { depois de preenchido. }\end{array}$ \\
\hline Elaboração & $\begin{array}{l}\text { Nome e assinatura do responsável pela elabo- } \\
\text { ração do formulário. }\end{array}$ \\
\hline Verificação/Aprovação & $\begin{array}{l}\text { Assinatura do responsável por verificar e } \\
\text { aprovar o conteúdo do formulário. }\end{array}$ \\
\hline & \\
\hline &
\end{tabular}

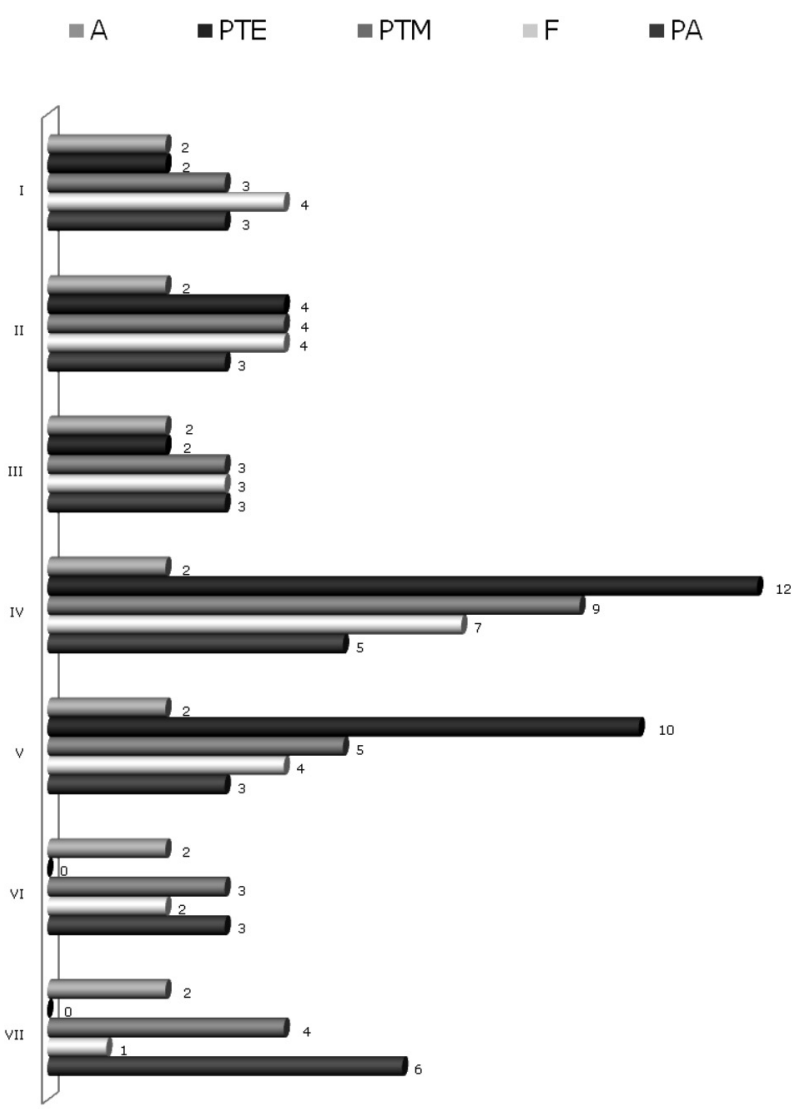

Figura 2. Distribuição dos documentos de BPL envolvidos na análise de resíduos de um herbicida em grãos de soja. $\boldsymbol{A}$ - Anexo. PTE - Procedimento Técnico de Equipamento. PTM - Procedimento Técnico de Metodologia. $\boldsymbol{F}$ - Formulário de Registro. PA - Procedimento Administrativo de uso da balança), 3 PTM (manuseio de amostras de sistema teste, documentação de dados brutos e método analítico), 2 PTE (balança, freezer e geladeira) e 2 anexos (matriz de competência e plano de estudo), perfazendo um total de 14 documentos.

Na etapa seguinte (processamento das amostras) foram utilizados 3 PA (inspeção de fase crítica, procedimento analítico e comunicação interna), 4 formulários de registros (processamento da amostra, uso da balança digital, controle do sistema teste e localização de amostras), 4 PTM (manuseio de amostras de sistema teste, processamento de amostra, método analítico e instruções sobre equipamentos), 4 PTE (balança digital, processador blendor, triturador de gelo e multiprocessador) e 2 anexos (matriz de competência e plano de estudo), perfazendo um total de 17 documentos.

Na etapa pesagem das amostras foram utilizados 3 PA (inspeção da fase crítica, treinamento de pessoal e comunicação interna), 3 formulários de registros (dados brutos da análise de resíduo, controle do sistema teste e registro de uso da balança), 3 PTM (método analítico, manuseio de amostra de sistema teste e documentação de dados brutos), 2 PTE (balança semianalítica, freezer e geladeira) e 2 anexos (plano de estudo e matriz de competência), perfazendo um total de 13 documentos.

Na etapa fortificação, extração e limpeza das amostras foram utilizados 5 PA (procedimento analítico, treinamento de pessoal, comunicação interna, inspeção de fase crítica e gestão de documentos), 7 formulários de registros (formulário de dados brutos de análise de resíduos, preparo de soluções, controle de resíduos, resinas, registro de uso da balança, uso do pHmetro e localização de amostras), 9 PTM (validação de métodos para análises de resíduos, documentação de dados brutos, instruções sobre equipamentos, insumos e suprimentos, limpeza de resinas, preparo de soluções, preparo de soluções padrões, método analítico e descarte de solventes), 12 PTE (balanças analíticas, homogeneizador, deionizador de água, centrífuga refrigerada, destilador de água, agitador magnético, freezer e geladeira, balança semianalítica, pHmetro, termo higrômetro, dissecador e evaporador rotatório) e 2 anexos (plano de estudo e matriz de competência), perfazendo um total de 35 documentos.

Na quinta etapa (análises cromatográficas) foram utilizados 3 PA (procedimento analítico, treinamento de pessoal e inspeção de fase crítica), 4 formulários de registros (uso do sistema de HPLC, avaliação da substância de referência, validação de método para análises de resíduos e formulário de dados brutos de análises de resíduos), 5 PTM (aquisição e processamento de dados, manutenção periódica do sistema de HPLC e controle de qualidade analítica, método analítico e descarte de solventes), 10 PTE (3 de bombas para HPLC, injetor automático, forno para coluna analítica, desgaseificador de fase móvel, detector de fluorescência, forno de reação pós-coluna, válvula de seis vias e controlador de sistema) e 2 anexos (plano de estudo e matriz de competência), perfazendo um total de 24 documentos.

Na penúltima etapa, quando ocorreu a análise dos dados obtidos da etapa anterior, foram utilizados 3 PA (documentação dos dados brutos, inspeção de fase crítica e gestão da documentação), 2 formulários de registros (planilha de cálculo e análise de conjunto de dados), 3 PTM (sistemas informatizados, documentação de dados brutos e controle de qualidade analítica) e 2 anexos (plano de estudo e matriz de competência), perfazendo um total de 10 documentos.

Como última etapa (elaboração do relatório) foram utilizados 6 PA (auditoria de dados e revisão de relatório, declaração da garantia da qualidade, gestão da documentação, treinamento de pessoal e procedimento analítico), um formulário de registro (relatório final), 4 PTM (método analítico, controle de qualidade analítica, elaboração de relatório técnico e documentação de dados brutos) e 2 anexos (plano de estudo e matriz de competência), perfazendo um total de 13 documentos. 
Essas etapas podem ser revistas resumidamente a seguir:

$\begin{array}{llcccccc} & \text { Analito } & \text { PA } & \text { F } & \text { PTM } & \text { PTE } & \text { A } & \text { TOTAL } \\ \text { I } & \text { Recebimento } & 3 & 4 & 3 & 2 & 2 & 14 \\ \text { II } & \text { Processamento } & 3 & 4 & 4 & 4 & 2 & 17 \\ \text { III } & \text { Pesagem } & 3 & 3 & 3 & 2 & 2 & 13 \\ \text { IV } & \text { Extração e limpeza } & 5 & 7 & 9 & 12 & 2 & 35 \\ \text { V } & \text { Análise cromatográfica } & 3 & 4 & 5 & 10 & 2 & 24 \\ \text { VI } & \text { Análise de dados } & 3 & 2 & 3 & 0 & 2 & 10 \\ \text { VII } & \text { Elaboração de relatório } & 6 & 1 & 4 & 0 & 2 & 13 \\ & \text { TOTAL } & 26 & 25 & 31 & 30 & 14 & 126\end{array}$

Independente da condução de um estudo específico a BPL exige uma rotina de controle para o laboratório reconhecido. Esse controle inclui o preenchimento de formulários de registros. A seguir encontram-se os controles efetuados no Laboratório de Análises de Resíduos do CPQBA/UNICAMP.

Sete formulários de registros (controle de cilindros de gases, controle de temperatura e umidade do laboratório, sendo necessárias duas leituras diárias, controle de temperatura dos freezers, controle de temperatura da geladeira, verificação de chuveiro e lava olhos e registro de medições e verificações da balança), 5 PTE (cilindro de gás, termo higrômetro, freezer e geladeira, balanças analítica e semianalítica), um PTM (instruções sobre equipamentos, suprimentos e insumos), 2 PA (treinamento de pessoal e comunicação interna) e um anexo (matriz de competência).

O reconhecimento do Laboratório de Análises de Resíduos do CPQBA/UNICAMP em conformidade com as normas de BPL foi obtido em fevereiro de 2010. Esse reconhecimento permitiu a utilização, em estudos realizados pelo laboratório, do selo emitido pelo INMETRO. Esse reconhecimento passa por um processo de renovação periódica, ao qual o laboratório já foi submetido. Em março de 2011 foi obtida a primeira confirmação do reconhecimento do laboratório.

\section{DISCUSSÃO}

No caso específico de um laboratório instalado dentro de uma universidade, as dificuldades no cumprimento das exigências da NITDICLA 035 podem ter um caráter variável, desde a falta de percepção dos benefícios até as restrições orçamentárias. Uma vez percebidos os benefícios, a maior dificuldade são os custos. A rotatividade de estudantes estagiários caracteriza-se como outra dificuldade, uma vez que necessitam ser conscientizados e treinados em BPL. Para minimizar essa dificuldade foi criado um programa de treinamento em BPL para todos os que iniciam as atividades no laboratório.

Estando sempre presente a preocupação na capacitação da equipe de funcionários, estagiários e bolsistas para executarem análises de contaminantes intencionais e não intencionais em produtos agrícolas, outros cursos se fazem necessários para uma qualificação, voltados às necessidades de cada um.

Objetivamente são necessários gastos com treinamentos, consultorias, contratação de pessoal, calibrações, adequações da infraestrutura, tempo de dedicação da equipe, obtenção do reconhecimento e manutenção do mesmo.
O tempo dedicado a implantar e implementar os princípios das normas de BPL foi de 4 anos envolvendo 6 pessoas.

A estrutura do laboratório para adequação à NIT-DICLA 035 envolveu um investimento perto de $\mathrm{R} \$ 500.000,00$. Desses, $20 \%$ foram oriundos da Reitoria, os outros $80 \%$ foram provenientes de recursos da própria Divisão.

O investimento em consultoria e cursos de capacitação foi de aproximadamente $\mathrm{R} \$ 9.000,00$. O custo aproximado junto ao INMETRO para se obter o reconhecimento foi de R $\$ 20.000,00$.

\section{CONCLUSÃO}

A implantação de BPL envolve um grande esforço e alocação de recursos. Uma vez iniciado de forma gradual e sistemática, o treinamento mostra a importância de cada atividade e necessidade de padronização das mesmas, principalmente se forem executadas por mais de uma pessoa. A princípio é necessário um gasto maior de tempo, comparando com uma situação em que não haja nenhuma padronização e confecção de procedimentos. Porém, a existência de um sistema solidificado e organizado traz a eliminação do retrabalho de uma atividade ou análise e, principalmente, o da repetição do treinamento. Sendo assim, se o mesmo não for registrado e não possuir uma base redigida não traz benefícios duradouros, acarretando um acréscimo das horas e prejudicando o funcionamento operacional do laboratório.

O extenso tempo dedicado, bem como o investimento financeiro para implantação e implementação da norma NIT-DICLA-035 do INMETRO vêm contribuindo de modo significativo para a melhora da qualidade no trabalho de rotina. Essa excelência acaba por beneficiar também as pesquisas no laboratório.

Os resultados positivos obtidos (implantação e implementação de BPL) somente foram possíveis graças à força de vontade e convicção de uma equipe coesa, bem como devido a uma infraestrutura material e econômica adequada e, ainda, pelo apoio da diretoria.

Como consequência de um sistema organizado de acordo com a BPL, o integrante da equipe passa a adotar uma postura profissional demonstrada pelo compromisso, trabalho em equipe e organização - requisitos indispensáveis tanto na pesquisa quanto no trabalho de rotina.

\section{REFERÊNCIAS}

1. Jürg, P. Em Good Laboratory Practice - The Why and the How; Mager, T., ed.; Springer Verlag: Berlin, Heldelberg, New York, 2005.

2. http://www.ibama.gov.br/bpl/home.htm, acessada em Abril 2011.

3. http://www.inmetro.gov.br/monitoramento_BPL/reconhecimento_BPL. asp, acessada em Abril 2011

4. http://www.eurachem.org/guides/pdf/CITAC\%20EURACHEM $\% 20$ GUIDE.pdf, acessada em Março 2008.

5. http://www.fapesp.br/boaspraticas/codigo_050911.pdf, acessada em Setembro 2011.

6. http://portal.anvisa.gov.br/wps/portal/anvisa/anvisa/home/agrotoxicotoxicologia acessada em Junho 2007.

7. Hornshuh, M. Jean. Em Good Laboratory Practice Standards: Applications for Field and Laboratory Studies; Garner, W. Y.; Barge, M. S.; Ussary, J. P., eds.; American Chemical Society: Washington, 1992, cap. 4. 